\title{
Tick-Borne Pathogens Shape the Native Microbiome Within Tick Vectors
}

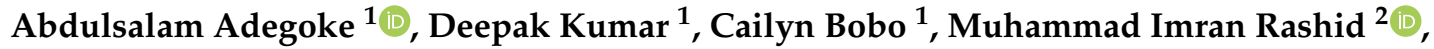 \\ Aneela Zameer Durrani ${ }^{3} \mathbb{D}$, Muhammad Sohail Sajid ${ }^{4}\left(\mathbb{D}\right.$ and Shahid Karim ${ }^{1, * \mathbb{D}}$ \\ 1 Center for Molecular and Cellular Biosciences, School of Biological, Environmental and Earth Sciences, \\ University of Southern Mississippi, Hattiesburg, MS 39406, USA; Abdulsalam.Adegoke@usm.edu (A.A.); \\ Deepak.Kumar@usm.edu (D.K.); Cailyn.Bobo@usm.edu (C.B.) \\ 2 Department of Parasitology, Faculty of Veterinary Science, The University of Veterinary and Animal Sciences, \\ Lahore 54000, Pakistan; imran.rashid@uvas.edu.pk \\ 3 Department of Clinical Medicine and Surgery, Faculty of Veterinary Science, The University of Veterinary \\ and Animal Sciences, Lahore 54000, Pakistan; aneela@uvas.edu.pk \\ 4 Department of Parasitology, Faculty of Veterinary Science, University of Agriculture, \\ Faisalabad 38000, Pakistan; drsohailuaf@hotmail.com \\ * Correspondence: Shahid.Karim@usm.edu; Tel.: +01-601-266-6232
}

Received: 13 July 2020; Accepted: 21 August 2020; Published: 25 August 2020

\begin{abstract}
Ticks are blood-feeding arthropods and transmit a variety of medically important viral, bacterial, protozoan pathogens to animals and humans. Ticks also harbor a diverse community of microbes linked to their biological processes, such as hematophagy, and hence affect vector competence. The interactions between bacterial and/or protozoan pathogens and the tick microbiome is a black-box, and therefore we tested the hypothesis that the presence of a protozoan or bacterial pathogen will alter the microbial composition within a tick. Hence, this study was designed to define the microbial composition of two tick species, Hyalomma (H.) anatolicum and Rhipicephalus (R.) microplus. We used a combination of PCR based pathogen (Anaplasma marginale and Theileria species) and symbiont (Wolbachia species) identification followed by metagenomic sequencing and comparison of the microbial communities in PCR positive and negative ticks. A total of 1786 operational taxonomic units was identified representing 25 phyla, 50 classes, and 342 genera. The phylum Proteobacteria, Firmicutes, Actinobacteriota, and Bacteroidota were the most represented bacteria group. Alpha and beta diversity were not significantly affected in the presence or absence of Theileria sp. and A. marginale as see with $H$. anatolicum ticks. Interestingly, bacterial communities were significantly reduced in Theileria sp. infected R. microplus ticks, while also exhibiting a significant reduction in microbial richness and evenness. Putting these observations together, we referred to the effect the presence of Theileria sp. has on R. microplus a "pathogen-induced dysbiosis". We also identify the presence of Plasmodium falciparum, the causative agent of human malaria from the microbiome of both $H$. anatolicum and R. microplus ticks. These findings support the presence of a "pathogen-induced dysbiosis" within the tick and further validation experiments are required to investigate how they are important in the vector competence of ticks. Understanding the mechanism of "pathogen-induced dysbiosis" on tick microbial composition may aid the discovery of intervention strategies for the control of emerging tick-borne infections.
\end{abstract}

Keywords: ticks; microbiome; Hyalomma anatolicum; Rhipicephalus microplus; Anaplasma marginale; Theileria sp.; Francisella; Wolbachia; Pakistan 


\section{Introduction}

Ticks are obligate, blood-feeding ectoparasites of vertebrate animals that depend on the host's blood for nutrition and reproduction. They elicit significant blood loss and also transmit disease-causing bacteria, viruses, and protozoa from one host to another, which makes them significant to public health. In Pakistan, livestock farming and production serve as one of the major drivers of the macro-economy as with the latest animal census showing an estimated population of 40 million, 47.8 million, 76.1 million, and 30.9 million buffalo, cattle, goats, and sheep, respectively, all contributing to an estimated $11.22 \%$ of the country's gross domestic product (GDP) [1].

Previously reported tick species infesting livestock in Pakistan includes both hard and soft tick species. In Pakistan, the most reported ticks found infesting livestock are hard ticks of the genus Haemaphysalis, Hyalomma, and Rhipicephalus, and soft ticks of the genus Argas and Ornithodoros [2-5]. Hyalomma and Rhipicephalus ticks are responsible for transmitting major tick-borne pathogens that affect livestock animals in Pakistan. Anaplasma marginale, Anaplasma centrale, Babesia bovis, and Babesia bigemina are which are known to cause cattle fever are transmitted by Rhipicephalus microplus ticks [6]. Tropical theileriosis, a small ruminant disease caused by Theileria annulata and the Crimean Congo hemorrhagic fever virus have been reported to be transmitted by Hyalomma anatolicum [6,7].

Ticks also harbor several distinct microbial communities, members of which have been shown to play an important role in tick biology. Analysis of the genome of such microbes has revealed specific regions coding for essential vitamins, most of which are lacking in the tick's blood meal [8-10], emphasizing their possible role as nutritional mutualists. Recent evidence also suggests that some members of these microbial communities can potentially interact with tick-borne pathogens both directly and indirectly. It was reported that removing the midgut bacteria of black-legged ticks Ixodes scapularis by feeding on antibiotic-treated mice impairs infection by Borrelia burgdorferi [11]. Another tick-borne pathogen, Anaplasma. phagocytophilum was shown to reduce the viability of the microbial population, thus facilitating its colonization of the I. scapularis midgut [12].

Along with the development in tick microbiome studies, adequate information exists on how tick-borne human pathogens shape the tick microbial communities to facilitate their colonization and subsequent transmission. While adequate research has been carried out on the microbiome of livestock infesting ticks, there is very little scientific understanding of the interactions that occurs between tick-transmitted pathogens and the tick microbial communities. Apart from Karim et al. [4], who reported important bacterial genera found in ticks from Pakistan, there is a general lack of research in understanding how specific bacterial or protozoan pathogens of animal origin shapes the microbiome of several tick vectors.

The main purpose of this study is to develop an understanding of changes that occurs in the microbial composition within a tick vector when such a tick acquires a bacterial or protozoan pathogen of animal source. The key research question of this study was whether or not the overall abundance and diversity of tick's microbial communities are reduced in the presence of a protozoan or bacteria pathogen. This study provides an exciting opportunity to advance our knowledge of tick microbiome and tick-borne pathogen interactions. A full discussion of the molecular mechanisms of reported interactions lies beyond the scope of this study. We will proceed to investigate specific microbial changes and interactions as part of our ongoing study. It is also beyond the scope of this study to examine whether host blood meal impacts microbial diversity.

\section{Materials and Methods}

\subsection{Tick Collection and Identification}

Fully engorged adult Hyalomma anatolicum and Rhipicephalus microplus ticks were randomly and carefully removed from livestock animals from four livestock producing regions in Pakistan

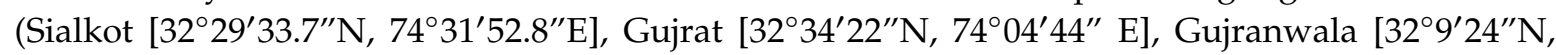


$74^{\circ} 11^{\prime} 24^{\prime \prime} \mathrm{E}$, and Sheikhupura [ $\left.\left.31^{\circ} 4247^{\prime \prime} \mathrm{N}, 73^{\circ} 58^{\prime} 41^{\prime \prime} \mathrm{E}\right]\right)$. This was done by careful removal of fully engorged ticks using tweezers with care been taken to keep the mouthparts intact. All ticks were kept in separate vials containing 70\% ethanol and details of the location, and the host was recorded. For this study, a total of 320 ticks were selected and shipped from Pakistan to the University of Southern Mississippi for further analysis using the U.S. Department of Agriculture's Animal and Plant Health Inspection Service (permit \# 11122050). Identification of ticks to the genus level was carried out by an expert taxonomist at the United States National Tick Collection (USNTC) according to the criteria used in previously published reports $[2,13,14]$. All stages were examined on an Olympus SZX16 stereoscopic microscope (Olympus Life Science, Center Valley, PA, USA). To further confirm morphological identification, ticks homogenates were subjected to molecular identification by amplifying the highly conserved $708 \mathrm{bp}$ mitochondrial Cytochrome Oxidase I gene (COI) [15]. The nucleotide accession numbers were MT876643, MT876644, and MT876645.

\subsection{Genomic DNA Extraction}

High-quality DNA was extracted from all the 320 ticks. Before DNA extraction, ticks were removed from the transport vials, cleaned using $100 \%$ ethanol, dried, and subsequently cleaned using a $10 \%$ sodium hypochlorite solution. Ticks were finally cleaned using distilled water and allowed to dry on a kimwipe paper. Homogenization of individual ticks was done mechanically, first by cutting ticks into smaller pieces, followed by complete disruption using an automated, hand-held homogenizer. The DNeasy Blood \& Tissue Kit (Qiagen, Germantown, MD, USA) was used to extract DNA from individual ticks with minor modification in the volume eluted $(30 \mu \mathrm{L})$. The DNA concentrations and quality were quantified using a nanodrop machine (Nanodrop One, Thermo Fisher Scientific, Pittsburgh, PA, USA) and DNA stored in $-20{ }^{\circ} \mathrm{C}$ till further needed.

\subsection{Detection of Pathogen and Endosymbiont}

To detect the presence of pathogens and endosymbiont of interest, we utilized a PCR based approach to amplify the $18 \mathrm{~S}$ rRNA gene of Theileria sp. [4], 16S rRNA gene of Anaplasma marginale [16], and GroEL gene of Wolbachia [17]. PCR positive DNAs were amplicon sequenced using both the forward and reverse primers and the partial sequences were subjected to NCBI BLAST program for further confirmation. Details of the primers, conditions, and amplicon sizes can be found in Table 1 . The Wolbachia sp. nucleotide accession numbers are MT881679, MT881680, MT881681, MT881682, MT881683, MT881684, MT881685, MT881686, MT881687, MT881688, MT881689, MT881690, MT881691, MT881692, MT881693, MT881694, MT881695, MT881696, MT881697, МT881698, MT881699, MT881700, MT881701 and MT881702.

Table 1. List of primers used in this study and their respective amplicon sizes.

\begin{tabular}{cccc}
\hline Target Genes & Primer Sequence $\left.\mathbf{( 5}^{\prime}-\mathbf{3}^{\prime}\right)$ & Amplicon Size (bp) & References \\
\hline Amar 16S-F & GGC GGT GAT CTG TAG CTG GTC TGA & $270 \mathrm{bp}$ & {$[16]$} \\
Amar 16S-R & GCC CAA TAA TTC CGA ACA ACG CTT & & \\
Theileria sp 18S-F & GGT AAT TCC AGC TCCAAT AG & $300 \mathrm{bp}$ & {$[4]$} \\
Theileria sp 18S-R & ACC AAC AAA ATA GAA CCA AAG TC & & {$[13]$} \\
16S rRNA 27F & AGR GTT TGA TCM TGG CTC AG & V1-V3 & {$[15]$} \\
16S rRNA 519R & GTN TTA CNG CGG CKG CTG & $708 \mathrm{bp}$ & {$[17]$} \\
COI-F & GGT CAA CAA ATC ATA AAG ATA TTG G & $800 \mathrm{bp}$ & \\
COI-R & TAA ACT TCA GGG TGA CCA AAA AAT CA & TGT ATT AGA TGA TAA CGT GC & \\
Wolbachia sp GroEL-F & CCA TTT GCA GAA ATT ATT GCA & & \\
Wolbachia sp GroEL-R & & &
\end{tabular}

\section{4. $16 S$ rRNA Library Preparation and Sequencing}

A total of 40 ticks ( $20 \mathrm{H}$. anatolicum and $20 \mathrm{R}$. microplus) were used for microbiome analysis. From each tick species, 5 Theileria sp. positive, 5 A. marginale positive, and 10 negative ticks were selected for $16 \mathrm{~S}$ rRNA library preparation and sequencing. The hypervariable V1-V3 region of the $16 \mathrm{~S}$ rRNA 
gene was PCR amplified using the forward primer 27F (5'-AGR GTT TGA TCM TGG CTC AG-3') and the reverse primer 519R ( $5^{\prime}$-GTN TTA CNG CGG CKG CTG-3') as outlined by the $16 \mathrm{~S}$ Illumina's MiSeq protocol (www.mrdnalab.com, Shallowater, TX, USA. Accessed on 11 July 2020). Briefly, PCR was performed using the HotStarTaq Plus Master Mix Kit (Qiagen, Germantown, Maryland, USA) under the following conditions: $94^{\circ} \mathrm{C}$ for $3 \mathrm{~min}$, followed by $30-35$ cycles of $94^{\circ} \mathrm{C}$ for $30 \mathrm{~s}, 53^{\circ} \mathrm{C}$ for $40 \mathrm{~s}$ and $72{ }^{\circ} \mathrm{C}$ for $1 \mathrm{~min}$, after which a final elongation step at $72{ }^{\circ} \mathrm{C}$ for $5 \mathrm{~min}$ was performed. After amplification, PCR products were electrophoresed in $2 \%$ agarose gel to determine the success of amplification and the relative intensity of bands. Multiple samples were pooled together in equal proportions based on their molecular weight and DNA concentrations. Pooled samples were purified using calibrated Ampure XP beads. Then the pooled and purified PCR product was used to prepare Illumina DNA library. Sequencing was performed at MR DNA (www.mrdnalab.com, Shallowater, TX, USA. Accessed on 11 July 2020) on a MiSeq following the manufacturer's guidelines.

\subsection{Sequence Analysis}

Sequence analysis was carried out using the Quantitative Insights into Microbial Ecology (QIIME 2) pipeline unless stated otherwise. Briefly, the processing of raw fastq files was demultiplexed. The Atacama soil microbiome pipeline was incorporated for quality control of demultiplexed paired-end reads using the DADA2 plugin as previously described [18]. Low-quality sequences were trimmed and filtered out, and subsequent merging of paired-end-reads was done ensuring 20 nucleotide overhang between forward and reverse reads. Chimeric sequences were removed from the sequence table.

Sequence alignment and subsequent construction of phylogenetic tree from representative sequences were performed using the MAFFT v. 7 and FasTree v. 2.1 plugins [19] Operational taxonomic assignment was performed using the qiime2 feature-classifier plugin v. 7.0, which was previously trained against the SILVA 138 database preclustered at $99 \%$. Tables representing operational taxonomic units (OTUs) and representative taxonomy were exported from $R$ and used for diversity metric analysis using the Microbiome Analyst web-based interface [20,21]. Raw data from this analysis were submitted deposited and assigned the GenBank BioProject number \#PRJNA600935.

\subsection{Alpha Diversity}

To establish whether alpha diversity differs across tick samples, reads were transformed and low abundance OTUs were filtered from the datasets. The Observed OTU metric was used to estimate species richness by identifying unique OTUs present across the tick groups, while the Shannon index was used to estimate both richness and evenness.

\subsection{Beta Diversity}

To compare the differences in the microbiome between tick groups, based on measures of distance or dissimilarity, dissimilarity matrix was generated from log-transformed sequence data and ordination of the plots was visualized using both the Principal Coordinates Analysis (PCoA) and the Nonmetric Multidimensional Scaling (NMDS). The Bray-Curtis distance matrix was used to visualize compositional differences in the microbiome across all groups.

\subsection{Statistical Analysis}

Statistical significance was inferred using the Mann-Whitney/Kruskal-Wallis method for alpha diversity and classical univariate comparison analysis, while the Permutational MANOVA (PERMANOVA) was used to test for the statistical significance of the dissimilarity measures. 


\section{Results}

\subsection{Pathogen and Symbiont Prevalence}

PCR analysis supported by amplicon sequencing and blast analysis of sequenced PCR product showed that $23(7.2 \%), 90(28.1 \%)$, and $3(0.9 \%)$ of the 320 individually tested ticks were positive for A. marginale, Theileria sp., and Wolbachia sp. (Table S1). We further determined the genetic relationship of the identified Wolbachia sp. and the tick species used with publicly available sequences from NCBI (Figures S1 and S2). The Wolbachia GroEL gene identified in ticks from this study shows high similarity to Wolbachia pipientis strain wAlbB-HN2016 and wAlbB-FL2016, with 99\% query cover and 99.61\% identity (Figure S3). The query cover and percentage identities of the COI sequences from this study were also compared to those previously deposited in the NCBI database (Figure S4).

\subsection{Bacteria $16 S$ rRNA Abundance Profile}

A total of 2,787,815 million reads paired-end reads were generated. Analysis of the demultiplexed paired-end-reads generated 2,787,815 reads which ranged from 36,124 to 123,736 with an average of 65,609 reads. After passing the sequences through quality filtering, 119,802 of the raw reads were non-chimeric which were subsequently used for taxonomic classification (Tables S2 and S3). Taxonomic classification using the SILVA reference base identified 472 OTUs generated from $R$. microplus ticks belonging to 10 phyla, 17 classes, and 146 genera. H. anatolicum had a total of 1314 OTUs representing 15 phyla, 33 classes, and 196 genera.

\subsection{Bacteria Relative Abundance}

The relative proportion of bacteria at different taxonomic classification was further analyzed in both tick species. Figure 1 presents the results obtained from the taxonomic classification of identified bacteria OTU at phylum, family, and genus taxonomic levels. Additional figures showing relative abundances of bacteria species in individual samples can be found (Figure S5A,B).

The phylum Proteobacteria, Firmicutes, Actinobacteriota, and Bacteroidota were all found to be present in H. anatolicum ticks. As shown in Figure 1A, the phylum Proteobacteria was found to be present at an abundance of $87.5 \%, 68 \%$, and $49 \%$ in Theileria sp. positive, uninfected and $A$. marginale positive $H$. anatolicum ticks, respectively, while Firmicutes (25\%) was only present in A. marginale infected ticks. Phylum level abundance in $R$. microplus ticks (Figure 1B) contrasts that shown in $H$. anatolicum. The entirety of the bacteria identified in Theileria sp. positive A. marginale belongs to the phylum Firmicutes (100\%), while both A. marginale positive and positive R. microplus shares similar bacteria phylum distribution representing Actinobacteria, Bacteroidota, and Proteobacteria (Figure 1B).

Francisellaceae $(37.5 \%)$ and Rickettsiales_fa (50\%) constituted to approximately $87.5 \%$ of the bacteria family identified within Theileria sp. positive $H$. anatolicum ticks. These bacteria families were also identified in uninfected ticks albeit at a much-reduced abundance (Figure 1C). Staphylococcaceae was identified at a relative abundance of $\sim 30 \%$ in $A$. marginale infected $H$. anatolicum. The family Anaplasmataceae (37.5\%) were identified at similar abundance in both $A$. marginale infected and uninfected $H$. anatolicum (Figure 1C). Distribution of the bacteria family in R. microplus ticks identified Bacillaceae at a $100 \%$ abundance in Theileria sp. positive $R$. microplus, while the family Corynebacteriaceae, Coxiellaceae, Flavobacteriaceae, Staphylococcaceae, and Weeksellaceae were detected in similar abundances in A. marginale positive and uninfected R. microplus (Figure 1D).

Similar differences in the bacteria abundances were further identified at the genus level which reflects those seen in the family and phylum. As can be seen from Figure 1E, five major genera; Acinetobacter, Anaplasma, Devosia, Norcadiopsis, and Sphingomonas which represents $87.5 \%$ of the bacteria were identified from $A$. marginale infected $H$. anatolicum ticks. The only genus of bacteria identified in Theileria sp. positive $H$. anatolicum ticks was Candidatus_Midichloria (51.5\%) and Francisella (36\%). The genus Candidatus_Midichloria and Francisella in addition to Ehrlichia, Hydrobacter, and Corynebacterium were identified in uninfected ticks (Figure 1E). Figure 1F shows similar bacterial 
composition at the genus level between uninfected and A. marginale positive $R$. microplus ticks, while the only identified genus in Theileria sp. positive $R$. microplus is Bacillus. These results of the bacteria abundance indicate that there is an association between the presences of Theileria sp. and how it shapes the bacteria composition of the two different tick species.
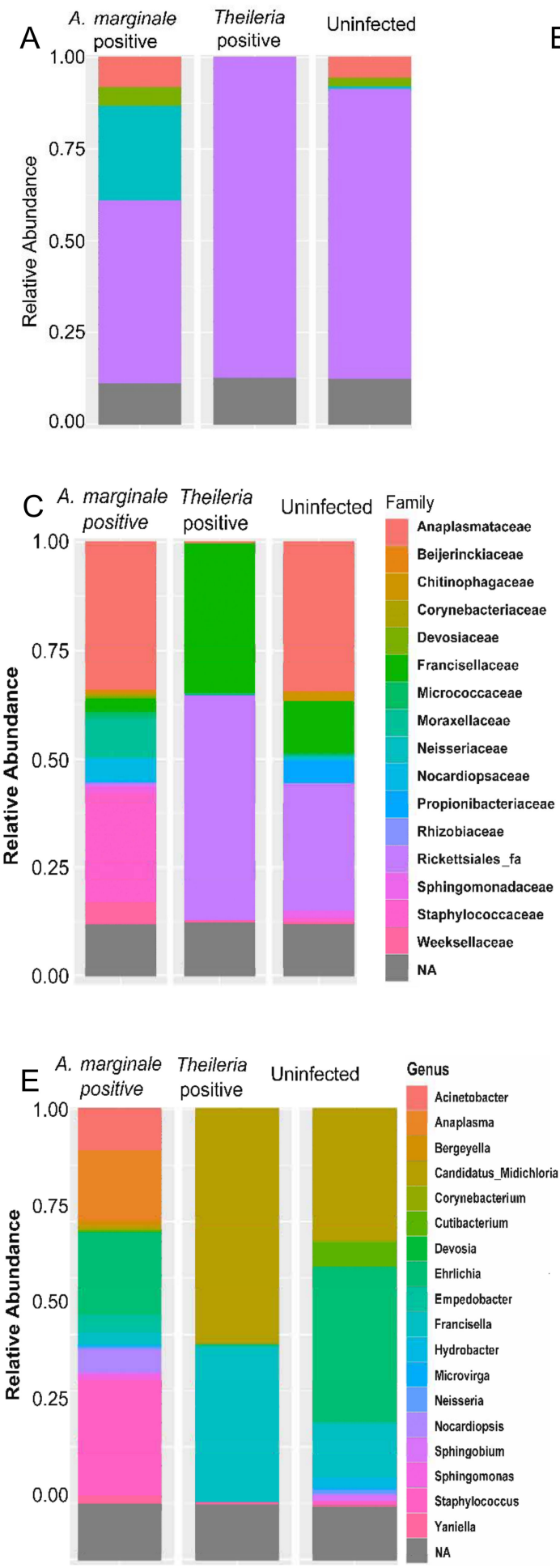
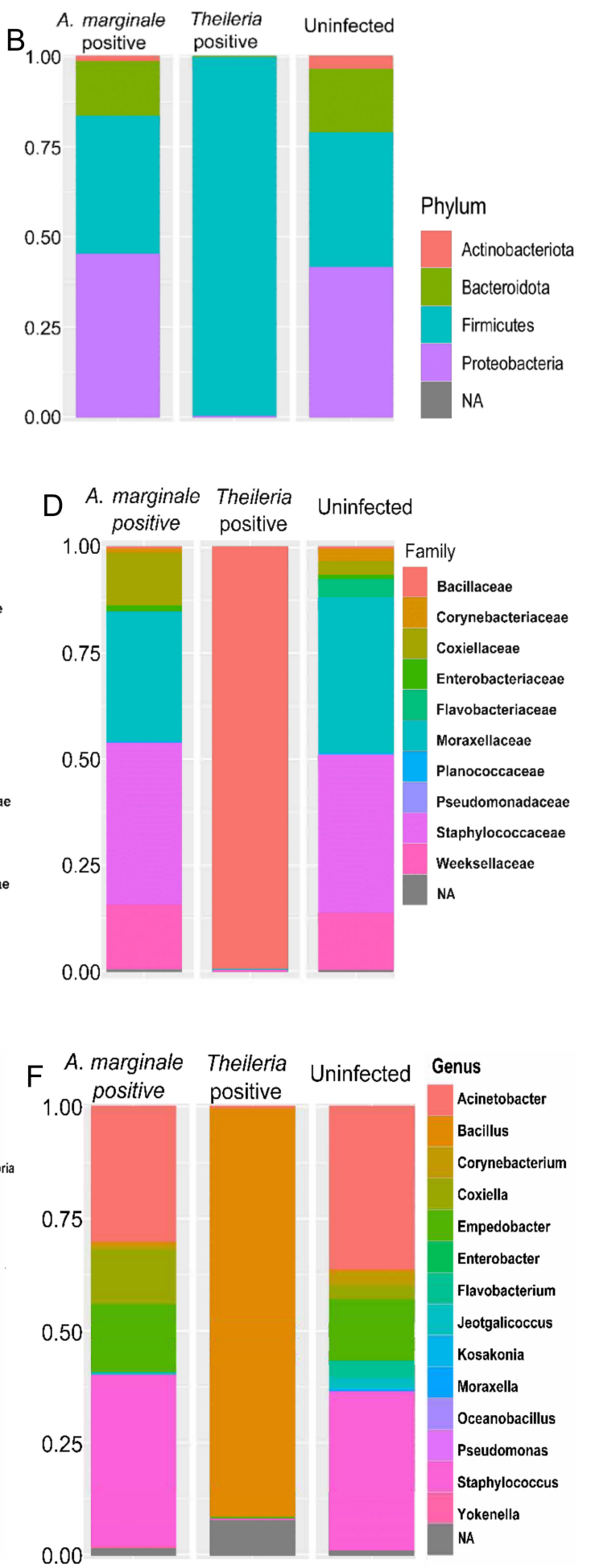

Figure 1. $16 \mathrm{~S}$ bacteria abundance profiles of H. anatolicum and R. microplus. (A,B) Bacteria abundance at phylum. (C,D). Bacteria abundance at the family level. (E,F) Bacteria abundance at the genus level. Hyalomma anatolicum (left panel), R. microplus (right panel). 


\subsection{Eukaryote $18 S$ rRNA Abundance}

We equally identified and compared eukaryote species in both ticks. Surprisingly, we detected the presence of Plasmodium falciparum in both tick groups, and Hepatozoon americanum in H. anatolicum ticks, both of which have a higher abundance in the Theileria sp. positive ticks (Figure 2A,B).
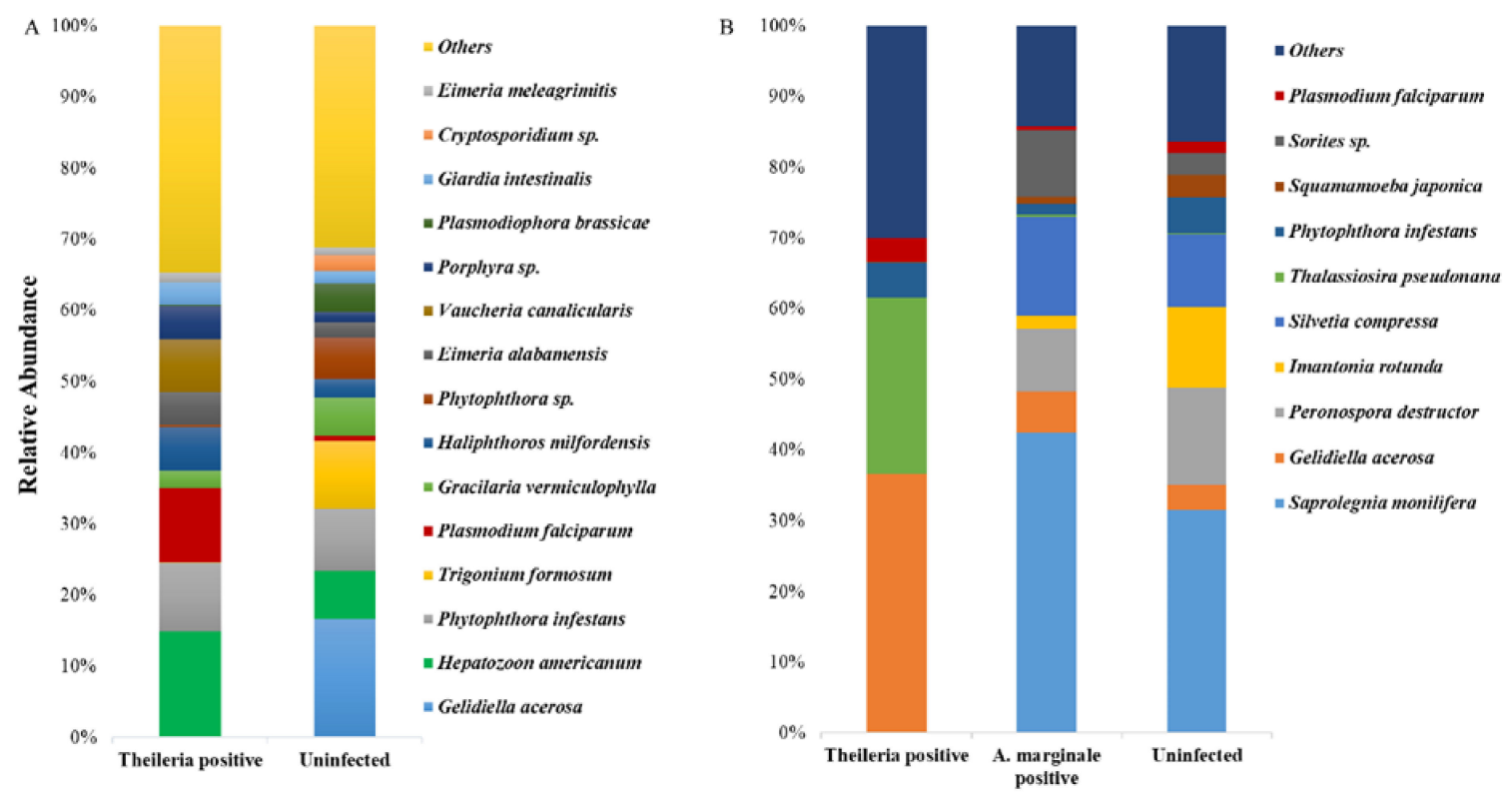

Figure 2. $18 \mathrm{~S}$ eukaryote abundance profiles of $H$. anatolicum and $R$. microplus. (A) The relative abundance of identified eukaryote species from Theileria sp. positive and uninfected $\mathrm{H}$. anatolicum ticks. (B) The relative abundance of identified eukaryote species from Theileria sp. positive, A. marginale positive, and uninfected R. microplus ticks.

\subsection{Microbial Richness and Evenness}

Microbial profile richness and evenness were estimated using the alpha diversity metrics observed OTUs and Shannon index. There was no significant difference between richness and evenness within the $H$. anatolicum ticks, irrespective of the PCR status (Figure S6A,B). Interestingly, R. microplus ticks showed comparable significant differences between Theileria sp. positive and uninfected ticks (Figure 3A,B). R. microplus ticks positive for Theileria sp. exhibited significantly reduced species richness and evenness index across all metrics used to analyze alpha diversity. 
A

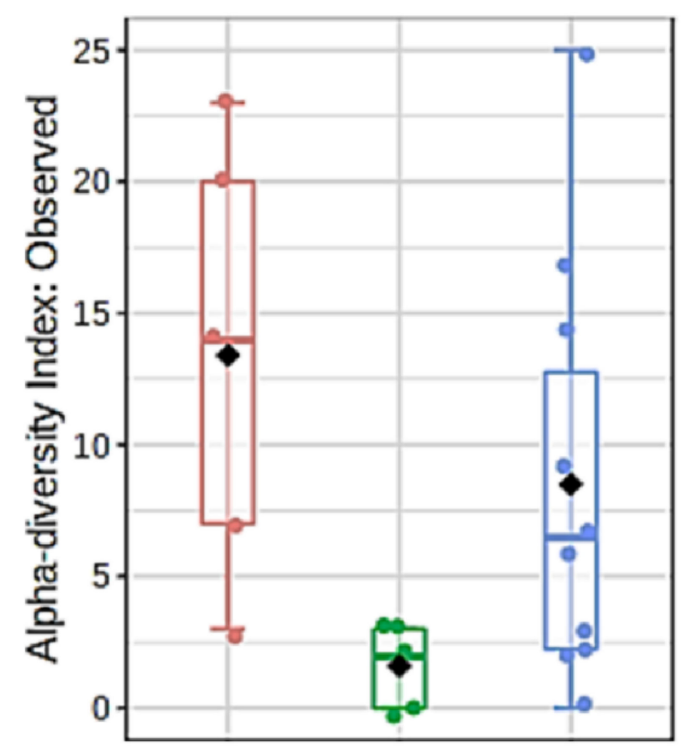

B

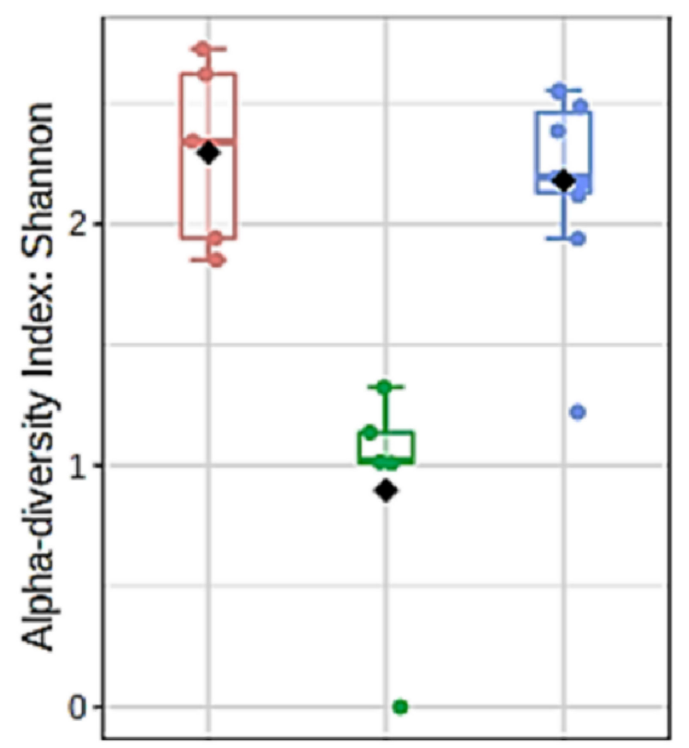

CLASS

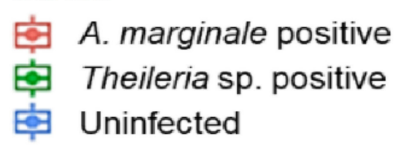

Figure 3. Alpha diversity analysis in R. microplus ticks. (A) Estimation of species richness using the observed operational taxonomic (OTUs) metrics (Kruskal-Wallis H-test, $\mathrm{df}=1, p$-value $=0.003$ ). (B) Estimation of species evenness using the Shannon diversity index (Kruskal-Wallis H-test, $\mathrm{df}=1$, $p$-value $=0.005$ ). Ticks found to be positive for Theileria sp. showed the least diversity using both measures.

\subsection{Microbial Similarity/Dissimilarity Patterns}

Differences in the microbial communities were analyzed using Bray-Curtis and Jaccard distance matrices. No significant observation was made in beta diversity across the $H$. anatolicum ticks (Figure S7A,B). Beta diversity was significantly different in $R$. microplus by both Bray-Curtis (PERMANOVA, F-value: 4.2171; R-squared: 0.33161; $p$-value $<0.005$; Figure 4A) and Jaccard (PERMANOVA, F-value: 3.2588; R-squared: 0.27714; $p$-value $<0.005$; Figure 4B). Non-metric multidimensional scaling (NMDS) plot of microbial communities further showed a distinct separation of Theileria sp. positive R. microplus ticks from both A. marginale and uninfected (Figure S8). 

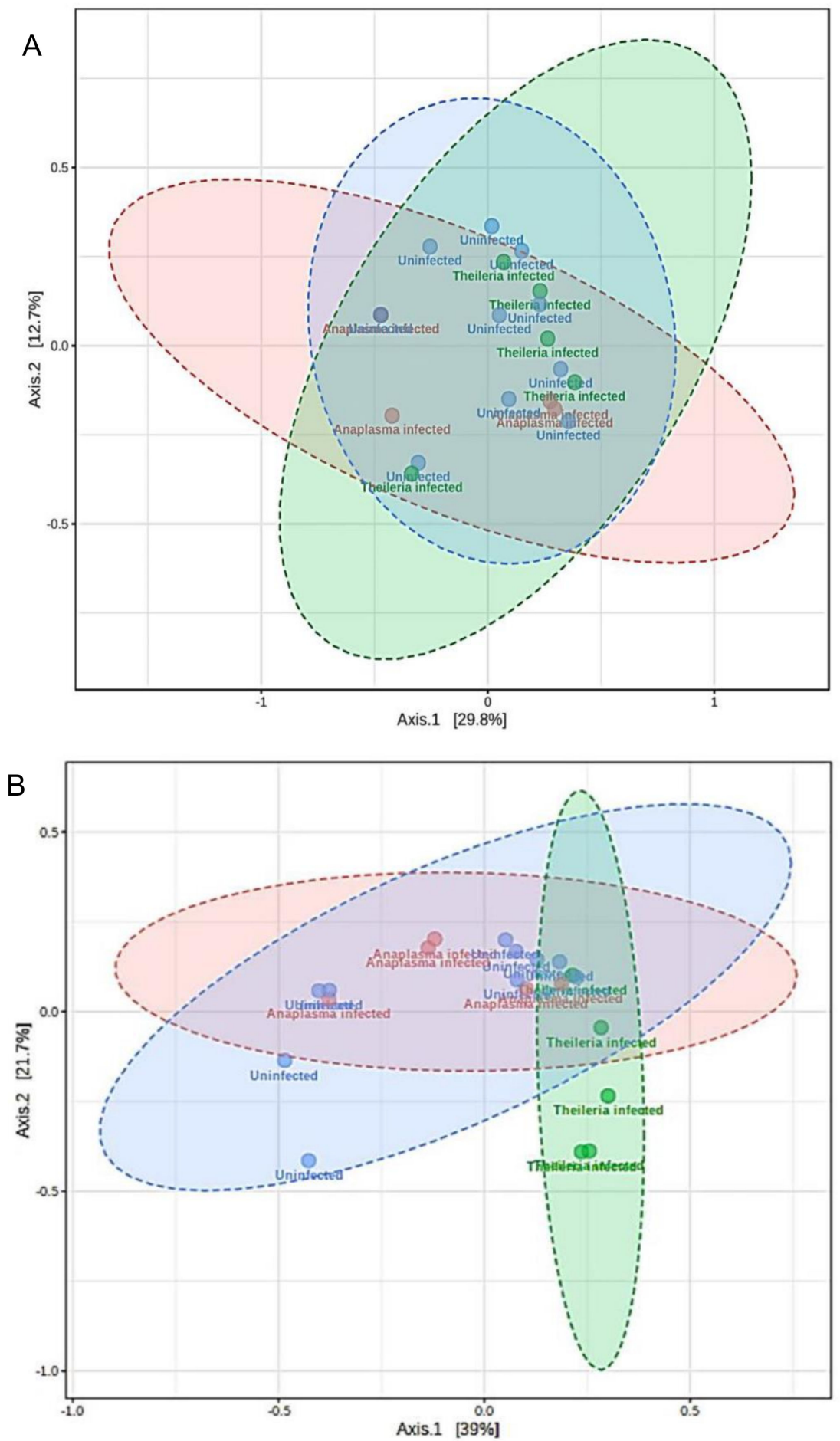

Figure 4. Estimation of differences in the microbial communities as a measure of Beta diversity analysis in R. microplus ticks. (A) Principal coordinate analysis (PCoA) of the Bray-Curtis distance matrix (PERMANOVA, F-value: 4.2171; R: 0.33161; $p$-value < 0.005). (B) Principal coordinate analysis (PCoA) Jaccard distance matrix (PERMANOVA, F-value: 3.2588; R: 0.27714; $p$-value $<0.005$ ).

\subsection{Community Profiling and Correlation Analysis of R. microplus Ticks}

To assess the extent to which highly abundant bacteria phylum and genus were represented in R. microplus ticks, we used a combination of pattern correlation and heat map analysis. A very strong positive correlation was seen between the presence of Bacillus and Theileria sp. positive R. microplus 
ticks (Figure 5). A similar observation was also seen in the heat map where the genus Bacillus shows the highest presence in Theileria sp. positive R. microplus ticks (Figure 6A).

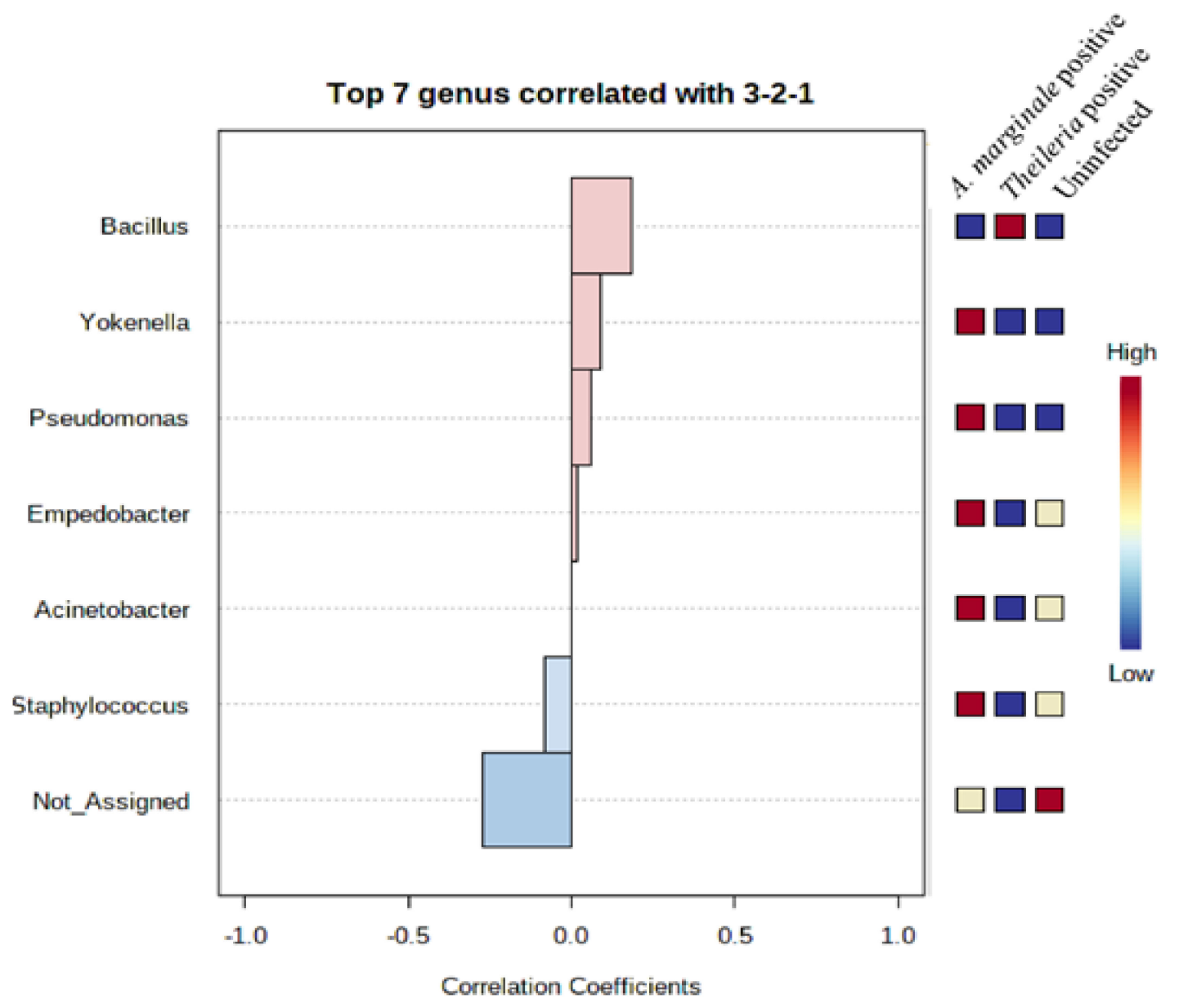

Figure 5. Pattern correlation analysis of top 7 bacteria genera in $R$. microplus ticks. Ticks positive with Theileria sp. showed a positive correlation with the presence of Bacillus.

To explore how top taxa differ, classical univariate statistical comparisons analysis was applied to identify bacterial genus that exhibits significant differences ( $t$-test/ANOVA) in their composition. Significant differences were observed in the abundance of the genus Acinetobacter, Staphylococcus, and Bacillus. Heat map analysis of OTU abundance was also estimated for H. anatolicum ticks, none of which was statistically significant (Figure S9).

In summary, the results of alpha and beta diversity as well as correlation analysis suggest that a strong association exists between Theileria sp., reduced alpha diversity metrics, and the distinct clustering separation exhibited by Theileria sp. positive R. microplus ticks. 


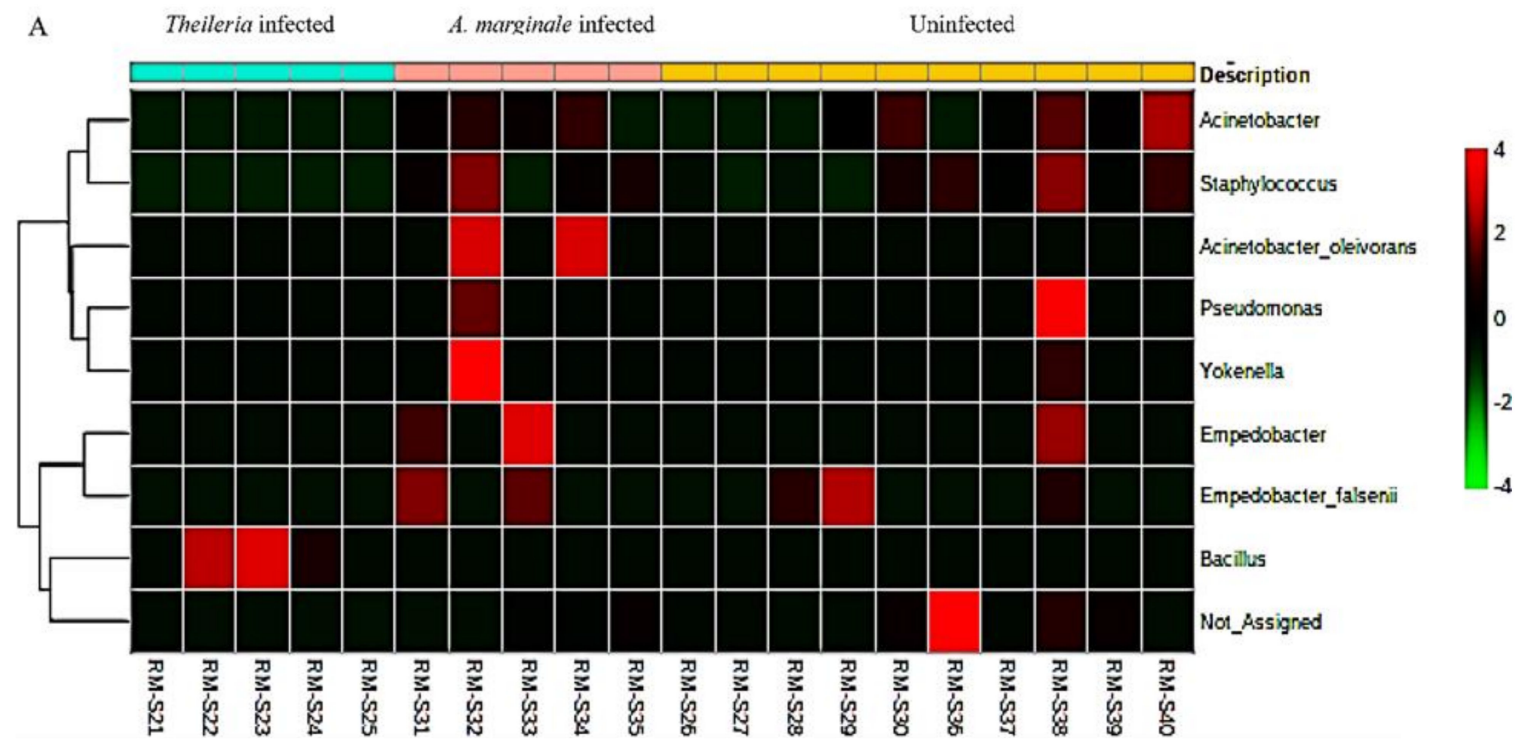

Acinetobacter

B

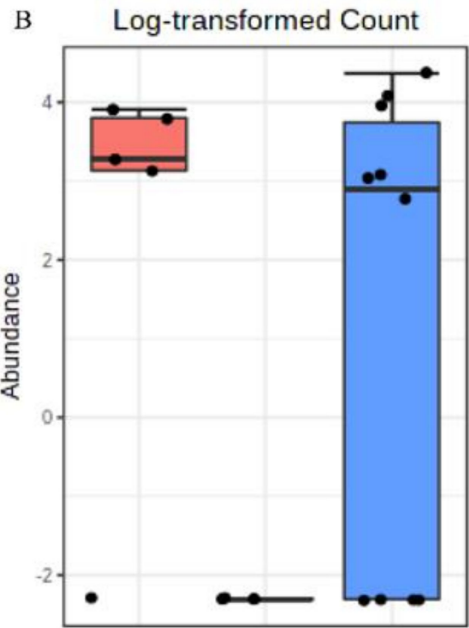

Description

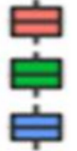

A. marginale positive

Theileria positive

Uninfected
Staphylococcus

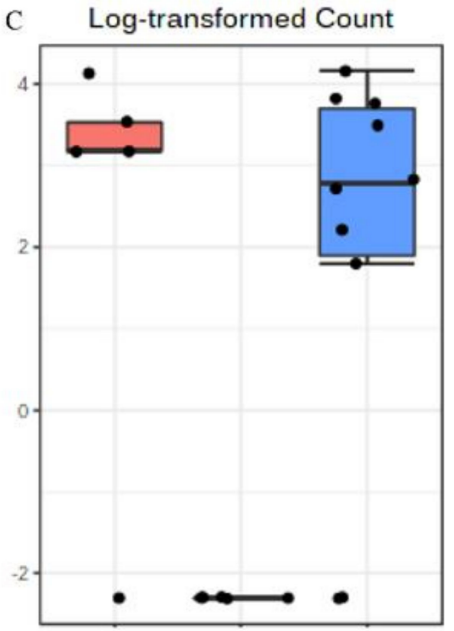

Bacillus

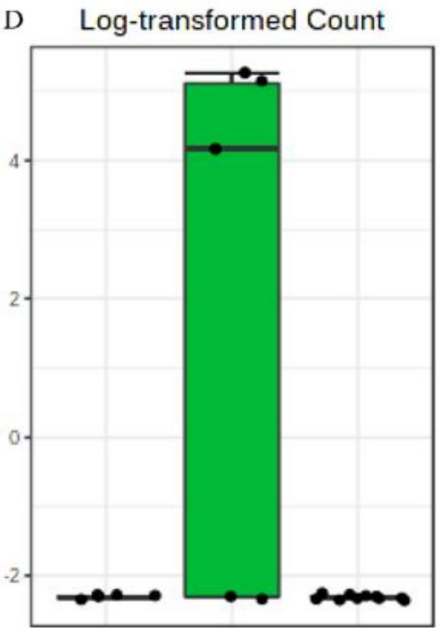

Figure 6. Heat map and classical univariate compositional analysis in R. microplus ticks. (A) Heat map correlation analysis between $A$. marginale positive, Theileria sp. positive and uninfected ticks. Log-transformed count of $(\mathbf{B})$ Acinetobacter $(\mathrm{FDR}=0.19277, \mathrm{df}=1, p$-value $=0.007),(\mathbf{C})$ Staphylococcus $(\mathrm{FDR}=0.19277, \mathrm{df}=1, p$-value $=0.007)$, and $(\mathrm{D})$ Bacillus $(\mathrm{FDR}=0.0002, \mathrm{df}=1, p$-value $=3.94 \mathrm{e}-223)$ in A. marginale positive, Theileria sp. positive and uninfected $R$. microplus ticks.

\section{Discussion}

The present study was designed to determine the changes that occur to the microbiome composition and diversity within the tick vectors when naturally infected with protozoan and bacterial tick-borne pathogens. Although many studies have detailed the plethora of interactions that occur between tick-transmitted pathogens and the microbiome of ticks such as endosymbionts and pathogen interactions [22], pathogen induction of antimicrobial production by the tick vector [12,23], only one of such studies compared the microbiome of pathogen-infected and uninfected ticks [24]. In this study, we tested field-collected H. anatolicum and R. microplus ticks for the presence of Theileria sp., 
Wolbachia sp., and A. marginale, and further compared the overall microbial distribution, richness, and diversity between Theileria sp. and $R$. microplus positive ticks. In the current study, the estimated percentage of Theileria sp. positive ticks was higher compared to those reported in previous studies for $H$. anatolicum, while $A$. marginale prevalence was similar to previous reports $[6,25,26]$.

The major bacteria phyla reported across all the tick groups irrespective of the PCR status were Proteobacteria, Bacteroidota, Firmicutes, and Actinobacteriota. These support observations from earlier tick microbiome studies $[4,11,27,28]$. Surprisingly, we identified a much lower number of bacterial reads and OTU from $R$. microplus ticks compared to H. anatolicum. This observation could be a function of the differences in the lifecycle of the two tick species. Rhipicephalus microplus is known as a one-host tick, whereas H. anatolicum ticks could spend their life cycle using 2-3 hosts. Spending different life stages on different animal hosts will likely expose the H. anatolicum ticks to a plethora of host skin microbial communities as well as host-blood [29] associated microbial communities. Some of the identified bacterial genera from $H$. anatolicum ticks such as Staphylococcus, Corynebacterium, Sphingomonas, and Cutibacterium have been previously shown to be common constituents of the skin microflora [29].

We also observed the presence of Theileria sp. was associated with an increased abundance of Candidatus_Midichloria and Francisella compared to the uninfected or A. marginale infected H. anatolicum. Candidatus_Midichloria and Francisella are obligate, vertically maintained endosymbionts in the phylum Proteobacteria. Candidatus_Midichloria belongs to the Alphaproteobacteria group of obligate intracellular bacteria first detected in Ixodes ricinus [30], while Francisella-like endosymbiont is a Gammaproteobacteria with widespread distribution in hard ticks [31]. It is interesting to compare these findings to an elegant observation made by Budachetri and colleagues [22] who proposed Candidatus_Midichloria mitochondrii as facilitating Rickettsia parkeri colonization of the Amblyomma maculatum tissues by protecting $R$. parkeri from the deleterious effect of reactive oxygen species. Our observations do require further experimental validation to understand the interaction between Theileria sp. and Candidatus_Midichloria within the tick vector.

The detection of Bacillus as the only bacteria genera identified from Theileria sp. positive R. microplus ticks was an unexpected, albeit important finding (Figure 1F). This observation was further validated by a significantly strong association between the presence of Theileria sp. and the Bacillus group of bacteria as seen in Figures 5 and 6A. We also found a higher abundance of the phylum Firmicutes (Supplementary Materials) and genus Bacillus was associated with Theileria sp. presence in R. microplus ticks (Figure 6). It is difficult to explain this result, but it might be related to the ability of Theileria sp. to interfere with the mammalian host's immune response by expressing proteins necessary for its transformation [32]. However, its impact on the tick microbiota has yet to be shown. Inhibition of important microbial metabolic pathways by Theileria sp.-associated proteins [33] could have led to a pathogen-associated dysbiosis. Bacillus ability to form spores when exposed to unfavorable physiological conditions would explain their exclusive presence in Theileria sp. positive $R$. microplus ticks.

We also observed that $R$. microplus ticks exhibited a significantly lower microbial diversity and composition when compared to H. anatolicum ticks. Within the R. microplus, those positive with Theileria sp. showed a significantly lesser amount of identified bacteria OTUs and a significantly reduced species richness and evenness (Figure 3A,B), while also displaying a different microbial composition as observed on the ordination plots (Figure 4A,B; Figure S5). These observations were consistent with those of Mann et al. [34], who reported that Trypanosoma cruzi infected kissing bugs exhibited a higher abundance of selected bacteria group, but not consistent with a study by Swei and Kwan [29] who reported that Ixodes pacificus ticks positive with the Lyme disease spirochete, Borrelia burgdorferi, had no significant differences in the microbiome richness and composition when compared to those not infected.

Another interesting finding from our study was the detection of the vertically transmitted Wolbachia sp. bacteria from a small number of the ticks used in this study. Wolbachia is an endosymbiont that has been identified in two-thirds of insects, including mosquitos [35], with known ability to 
interfere with their host's reproduction [36] through a series of physiological alterations, one of which is cytoplasmic incompatibility [37]. Studies showed the presence of Wolbachia in tick species has associated it with a form of hyperparasitism where Wolbachia was found infecting another parasitoid that parasitizes the ticks [38,39].

The unexpected identification of Plasmodium falciparum from ticks used in this study is an unpredicted finding. Pakistan is a malaria-endemic country and we observed an increase in the relative abundance of Plasmodium falciparum in Theileria sp. positive H. anatolicum when compared to the uninfected ticks (Figure 2A,B). It seems possible that this could have occurred from the ticks accidentally feeding on a P. falciparum-infected human host. While this is a possibility in multi-host ticks as seen in Hyalomma species, this is highly an unlikely occurrence in $R$. microplus which is a one-host tick. These results, therefore, need to be interpreted with caution as ticks are not reservoirs or competent vectors of $P$. falciparum.

This finding, while preliminary, suggests that the presence of Theileria sp. within R. microplus ticks reduces the overall microbial diversity which we proposed as "pathogen-induced dysbiosis". The mechanism behind this phenomenon could be induced by Theileria sp. factors in an attempt to colonize the tick vector, or it could be a result of the innate immune response mounted by the tick. These findings may help us to understand the intricate interplay of the pathogen-microbiome-vector interactions. However, more research on these observed interactions needs to be undertaken before the association between microbiome dysbiosis and the presence of a pathogen can be drawn.

\section{Conclusions}

The present study was designed to determine the effect of bacteria and protozoan pathogen on the microbiome of field-collected ticks. Using a combination of PCR based assay and 16S rRNA sequencing, we investigated how the presence of Theileria sp. and A. marginale shapes the overall microbiome of both $H$. anatolicum and $R$. microplus ticks. We reported a strong association between the presence of Theileria sp. and a completely reduced microbial diversity and abundance in $R$. microplus ticks. This study established the extent of the diversity of the microbial community within two important tick species from Pakistan and revealed the presence of Theileria sp., A. marginale, and additional pathogenic bacteria that could be of public health significance. A limitation of this study was the difficulty in obtaining tissue samples of ticks, as they were field collected. Future tick developmental and tissue-specific studies will generate new insights into specific interactions between tick-borne pathogens and their associated microbiomes.

Supplementary Materials: The following are available online at http://www.mdpi.com/2076-2607/8/9/1299/s1. Figure S1: Phylogenetic analysis of amplified COI sequences of $R$. microplus and $H$. anatolicum ticks with other arthropod's COI genes from NCBI, Figure S2: Genetic relationship of identified Wolbachia GroEL genes compared with selected sequences from NCBI, Figure S3: Blast results of the percentage identity and query cover of Wolbachia GroEL sequence from the current study when compared to previously deposited GroEL sequences from NCBI, Figure S4: Blast results of the percentage identity and query cover of Hyalomma anatolicum and Rhipicephalus microplus COI sequence from the current study when compared to previously deposited COI sequences from NCBI, Figure S5: Relative abundances of bacteria species from individual tick samples used in this study, Figure S6. Alpha diversity analysis in R. microplus ticks, Figure S7: Visualization of differences in the microbial communities using Principal coordinate analysis (PCoA) of Bray_Curtis and Jaccard distance matrix, Figure S8: Non-metric multidimensional scaling (NMDS) plot of Bray_Curtis and Jaccard distance matrix, Table S1: Total number of ticks used in this study and the percentage of those identified with A. marginale, Theileria, and Wolbachia based on PCR analysis and amplicon sequencing, Table S2: Total number of reads that used in the analysis after filtering and removal of chimeras in H. anatolicum ticks, Table S3: Total number of reads that used in the analysis after filtering and removal of chimeras in R. microplus ticks.

Author Contributions: Conceptualization: S.K., data curation: A.A., D.K. and S.K.; formal analysis: A.A., C.B., D.K. and S.K.; funding acquisition: S.K., M.I.R., A.Z.D. and M.S.S.; investigation: A.A., D.K., M.I.R., A.Z.D., M.S.S. and S.K.; methodology: A.A. and S.K.; project administration: S.K., M.I.R., A.Z.D. and M.S.S.; Resources: S.K., M.I.R., A.Z.D. and M.S.S.; supervision: S.K.; validation: A.A., D.K. and S.K.; visualization: A.A., D.K. and S.K.; writing—original draft: A.A. and S.K.; and writing-review and editing: S.K. All authors have read and agreed to the published version of the manuscript. 
Funding: This research was funded by a Pakistan-US Science and Technology Cooperation Program award (US Department of States; the Mississippi INBRE (an institutional Award (IDeA) from the National Institute of General Medical Sciences of the National Institutes of Health under award P20GM103476). Cailyn Bobo was supported by the Eagle SPUR award from the USM Drapeau Center for Undergraduate Research. The funders played no role in the study design, data collection, and analysis, decision to publish, or preparation of the manuscript.

Conflicts of Interest: The authors declare that they have no competing interests.

Availability of data and material: The datasets supporting the conclusion of this article are included within the article and its additional files. Raw data are available from the corresponding author upon request.

\section{References}

1. Ministry of Finance, Government of Pakistan. Available online: http://www.finance.gov.pk/survey_1819.html (accessed on 11 July 2020).

2. Hoogstraal, H.; Varma, M.G.R. Haemaphysalis cornupunctata sp. n. and H. kashmirensis sp. n. from Kashmir, with Notes on H. sundrai Sharif and H. sewelli Sharif of India and Pakistan (Ixodoidea, Ixodidae). J. Parasitol. 1962, 48, 185. [CrossRef] [PubMed]

3. Robertson, R.G.; Wisseman, C.L.; Traub, R. Tick-borne rickettsiae of the spotted fever group in West Pakistan: I. Isolation of strains from ticks in different habitats. Am. J. Epidemiol. 1970, 92, 382-394. [CrossRef] [PubMed]

4. Karim, S.; Budachetri, K.; Mukherjee, N.; Williams, J.; Kausar, A.; Hassan, M.J.; Adamson, S.; Dowd, S.E.; Apanskevich, D.; Arijo, A.; et al. A study of ticks and tick-borne livestock pathogens in Pakistan. PLoS Negl. Trop. Dis. 2017, 11, e0005681. [CrossRef]

5. Zeb, J.; Szekeres, S.; Takács, N.; Kontschán, J.; Shams, S.; Ayaz, S.; Hornok, S. Genetic diversity, piroplasms and trypanosomes in Rhipicephalus microplus and Hyalomma anatolicum collected from cattle in northern Pakistan. Exp. Appl. Acarol. 2019, 79, 233-243. [CrossRef]

6. Jabbar, A.; Abbas, T.; Sandhu, Z.U.D.; Saddiqi, H.A.; Qamar, M.F.; Gasser, R.B. Tick-borne diseases of bovines in Pakistan: Major scope for future research and improved control. Parasites Vectors 2015, 8, 283. [CrossRef]

7. Atif, M.; Saqib, A.; Ikram, R.; Sarwar, M.R.; Scahill, S. The reasons why Pakistan might be at high risk of Crimean Congo hemorrhagic fever epidemic; a scoping review of the literature. Virol. J. 2017, 14, 63. [CrossRef]

8. Sjödin, A.; Svensson, K.; Ohrman, C.; Ahlinder, J.; Lindgren, P.; Duodu, S.; Johansson, A.; Colquhoun, D.J.; Larsson, P.; Forsman, M. Genome characterization of the genus Francisella reveals insight into similar evolutionary paths in pathogens of mammals and fish. BMC Genomics 2012, 13,268. [CrossRef]

9. Gottlieb, Y.; Lalzar, I.; Klasson, L. Distinctive genome reduction rates revealed by genomic analyses of two Coxiella-like endosymbionts in ticks. Genome Biol. Evol. 2015, 7, 1779-1796. [CrossRef]

10. Smith, T.A.; Driscoll, T.; Gillespie, J.J.; Raghavan, R. A Coxiella-like endosymbiont is a potential vitamin source for the lone star tick. Genome Biol. Evol. 2015, 7, 831-838. [CrossRef] [PubMed]

11. Narasimhan, S.; Rajeevan, N.; Liu, L.; Zhao, Y.O.; Heisig, J.; Pan, J.; Eppler-Epstein, R.; Deponte, K.; Fish, D.; Fikrig, E. Gut microbiota of the tick vector Ixodes scapularis modulate colonization of the Lyme disease spirochete. Cell Host Microbe 2014, 15, 58-71. [CrossRef] [PubMed]

12. Abraham, N.M.; Liu, L.; Jutras, B.L.; Yadav, A.K.; Narasimhan, S.; Gopalakrishnan, V.; Ansari, J.M.; Jefferson, K.K.; Cava, F.; Jacobs-Wagner, C.; et al. Pathogen-mediated manipulation of arthropod microbiota to promote infection. Proc. Natl. Acad. Sci. USA 2017, 114, E781-E790. [CrossRef] [PubMed]

13. Dowd, S.E.; Callaway, T.R.; Wolcott, R.D.; Sun, Y.; McKeehan, T.; Hagevoort, R.G.; Edrington, T.S. Evaluation of the bacterial diversity in the feces of cattle using $16 \mathrm{~S}$ rDNA bacterial tag-encoded FLX amplicon pyrosequencing (bTEFAP). BMC Microbiol. 2008, 8, 125. [CrossRef] [PubMed]

14. Mukherjee, N.; Beati, L.; Sellers, M.; Burton, L.; Adamson, S.; Robbins, R.G.; Moore, F.; Karim, S. Importation of exotic ticks and tick-borne spotted fever group rickettsiae into the United States by migrating songbirds. Ticks Tick-borne Dis. 2014, 5, 127-134. [CrossRef] [PubMed]

15. Dougherty, L.; Li, J. Molecular phylogeny and morphological distinctions of two popular bivalves, Ctenoides scabei and Ctenoides mitis. J. Mar. Boil. 2017, 2017, 1-9. [CrossRef]

16. Kundave, V.; Ram, H.; Banerjee, P.S.; Garg, R.; Mahendran, K.; Ravikumar, G.; Tiwari, A.K. Development of multiplex PCR assay for concurrent detection of tick-borne haemoparasitic infections in bovines. Acta Parasitol. 2018, 63, 759-765. [CrossRef] [PubMed] 
17. Masui, S.; Sasaki, T.; Ishikawa, H. groE-Homologous Operon of Wolbachia, an Intracellular Symbiont of Arthropods: A New Approach for Their Phylogeny. Zool. Sci. 1997, 14, 701-706. [CrossRef]

18. Callahan, B.J.; McMurdie, P.J.; Rosen, M.J.; Han, A.W.; Johnson, A.J.A.; Holmes, S.P. DADA2: High-resolution sample inference from Illumina amplicon data. Nat. Methods 2016, 13, 581-583. [CrossRef]

19. Price, M.N.; Dehal, P.S.; Arkin, A.P. FastTree 2-Approximately maximum-likelihood trees for large alignments. PLoS ONE 2010, 5, e9490. [CrossRef]

20. Chong, J.; Liu, P.; Zhou, G.; Xia, J. Using Microbiome Analyst for comprehensive statistical, functional, and meta-analysis of microbiome data. Nat. Protoc. 2020, 15, 799-821. [CrossRef]

21. Dhariwal, A.; Chong, J.; Habib, S.; King, I.L.; Agellon, L.B.; Xia, J. Microbiome Analyst: A web-based tool for comprehensive statistical, visual and meta-analysis of microbiome data. Nucleic Acids Res. 2017, 45, W180-W188. [CrossRef]

22. Budachetri, K.; Kumar, D.; Crispell, G.; Beck, C.; Dasch, G.; Karim, S. The tick endosymbiont Candidatus Midichloria mitochondrii and selenoproteins are essential for the growth of Rickettsia parkeri in the Gulf Coast tick vector. Microbiome 2018, 6, 141. [CrossRef] [PubMed]

23. Narasimhan, S.; Schuijt, T.J.; Abraham, N.M.; Rajeevan, N.; Coumou, J.; Graham, M.; Robson, A.; Wu, M.-J.; Daffre, S.; Hovius, J.W.; et al. Modulation of the tick gut milieu by a secreted tick protein favors Borrelia burgdorferi colonization. Nat. Commun. 2017, 8, 1-17. [CrossRef] [PubMed]

24. Fryxell, R.T.T.; DeBruyn, J.M. The Microbiome of Ehrlichia-infected and uninfected lone star ticks (Amblyomma americanum). PLoS ONE 2016, 11, e0146651.

25. Durrani, A.Z.; Kamal, N. Identification of ticks and detection of blood protozoa in Friesian cattle by polymerase chain reaction test and estimation of blood parameters in district Kasur, Pakistan. Trop. Anim. Heal. Prod. 2008, 40, 441-447. [CrossRef]

26. Rehman, A.; Nijhof, A.M.; Sauter-Louis, C.; Schauer, B.; Staubach, C.; Conraths, F.J. Distribution of ticks infesting ruminants and risk factors associated with high tick prevalence in livestock farms in the semi-arid and arid agro-ecological zones of Pakistan. Parasites Vectors 2017, 10, 1-15. [CrossRef]

27. Budachetri, K.; Browning, R.E.; Adamson, S.W.; Dowd, S.E.; Chao, C.-C.; Ching, W.-M.; Karim, S. An insight into the microbiome of the Amblyomma maculatum (Acari: Ixodidae). J. Med. Entomol. 2014, 51, 119-129. [CrossRef]

28. Travanty, N.V.; Ponnusamy, L.; Kakumanu, M.L.; Nicholson, W.L.; Apperson, C.S. Diversity and structure of the bacterial microbiome of the American dog tick, Dermacentor variabilis, is dominated by the endosymbiont Francisella. Symbiosis 2019, 79, 1-12. [CrossRef]

29. Swei, A.; Kwan, J.Y. Tick microbiome and pathogen acquisition altered by host blood meal. ISME J. 2017, 11, 813-816. [CrossRef]

30. Epis, S.; Mandrioli, M.; Genchi, M.; Montagna, M.; Sacchi, L.; Pistone, D.; Sassera, D. Localization of the bacterial symbiont Candidatus Midichloria mitochondrii within the hard tick Ixodes ricinus by whole-mount FISH staining. Ticks Tick-borne Dis. 2013, 4, 39-45. [CrossRef]

31. Dergousoff, S.J.; Chilton, N.B. Association of different genetic types of Francisella-like organisms with the rocky mountain wood tick (Dermacentor andersoni) and the American Dog Tick (Dermacentor variabilis) in Localities Near Their Northern Distributional Limits. Appl. Environ. Microbiol. 2011, 78, 965-971. [CrossRef]

32. Chakraborty, S.; Roy, S.; Mistry, H.U.; Murthy, S.; George, N.; Bhandari, V.; Sharma, P. Potential sabotage of host cell physiology by apicomplexan parasites for their survival benefits. Front. Immunol. 2017, 8. [CrossRef] [PubMed]

33. Bahia, A.C.; Oliveira, J.H.M.; Kubota, M.S.; Araújo, H.R.C.; Lima, J.B.P.; Ríos-Velásquez, C.M.; Lacerda, M.V.G.; De Oliveira, P.L.; Traub-Csekö, Y.M.; Pimenta, P.F.P. The Role of Reactive Oxygen Species in Anopheles aquasalis Response to Plasmodium vivax Infection. PLoS ONE 2013, 8, 1-10. [CrossRef] [PubMed]

34. Mann, A.E.; Mitchell, E.A.; Zhang, Y.; Curtis-Robles, R.; Thapa, S.; Hamer, S.A.; Allen, M.S. Comparison of the bacterial gut microbiome of North American Triatoma spp. With and without Trypanosoma cruzi. Front. Microbiol. 2020, 11, 364. [CrossRef] [PubMed]

35. Hilgenboecker, K.; Hammerstein, P.; Schlattmann, P.; Telschow, A.; Werren, J.H. How many species are infected with Wolbachia? A statistical analysis of current data. FEMS Microbiol. Lett. 2008, 281, 215-220. [CrossRef]

36. Sicard, M.; Bonneau, M.; Weill, M. Wolbachia prevalence, diversity, and ability to induce cytoplasmic incompatibility in mosquitoes. Curr. Opin. Insect Sci. 2019, 34, 12-20. [CrossRef] [PubMed] 
37. Stouthamer, R.; Breeuwer, J.A.J.; Hurst, G.D.D. Wolbachia Pipientis: Microbial manipulator of arthropod reproduction. Annu. Rev. Microbiol. 1999, 53, 71-102. [CrossRef]

38. Plantard, O.; Bouju-Albert, A.; Malard, M.-A.; Hermouet, A.; Capron, G.; Verheyden, H. Detection of Wolbachia in the tick Ixodes ricinus is due to the presence of the Hymenoptera endoparasitoid Ixodiphagus hookeri. PLoS ONE 2012, 7, e30692. [CrossRef]

39. Tijsse-Klasen, E.; Braks, M.; Scholte, E.J.; Sprong, H. Parasites of vectors-Ixodiphagus hookeri and its Wolbachia symbionts in ticks in The Netherlands. Parasites Vectors 2011, 4, 228. [CrossRef]

(C) 2020 by the authors. Licensee MDPI, Basel, Switzerland. This article is an open access article distributed under the terms and conditions of the Creative Commons Attribution (CC BY) license (http://creativecommons.org/licenses/by/4.0/). 